\title{
Surgical Airway Education among Anesthesia Trainees: A Cohort Study
}

\author{
Russel Kahmke ${ }^{*}$, Matthew G Crowson ${ }^{2}$, Suraj Yalamuri ${ }^{2}$, Bryant W Stolp ${ }^{2}$, Liana Puscas ${ }^{1}$ and Charles R Woodard $^{1}$ \\ ${ }^{1}$ Division of Otolaryngology, Head and Neck Surgery, Department of Surgery, Duke University Health System, Durham, NC, USA \\ ${ }^{2}$ Department of Anesthesiology, Duke University Health System, Durham, NC, USA
}

\begin{abstract}
Purpose: Emergency invasive airway access is necessary when a provider encounters a "can't intubate, can't ventilate" situation where non-invasive techniques fail. Regardless of advanced airway training, a cricothyrotomy is easier, faster, and associated with less bleeding when compared to a tracheotomy. The knowledge, confidence, and procedural ability of anesthesia residents in performing a cricothyrotomy were assessed.

Methods: A 2014 IRB approval was obtained. After successful intubation, anesthesia residents were provided a brief clinical vignette where the only viable option was emergency invasive airway access. They were given a marking pen and asked to perform a mock cricothyrotomy. A multimedia educational program illustrating anatomy, procedural details, and alternate procedures was subsequently provided. At least 4 weeks later, the residents were again presented with the opportunity to participate.

Results: Nine residents consented to participate before and 18 after the educational component. There was no difference between the patients used in each group. When adjusted for number of years in training and size of the patient, there was no difference in accuracy between the pre- and post- intervention groups. The educational program produced a statistically significant procedural time improvement for all participants. There was improvement in time for normal and overweight patients, with a more significant improvement for overweight patients. Both junior and senior level residents were faster without statistical difference between the two.

Conclusions: A knowledge and confidence deficit in emergency invasive airway management exists; directed education results in improved procedural timing, especially with overweight patients.
\end{abstract}

\section{Introduction}

The American Society of Anesthesiologists developed a difficult airway algorithm to assist in the management of patients with complex airway needs [1]. When a provider encounters a "can't intubate, can't ventilate" situation where non-invasive techniques fail to provide ventilation, emergency invasive airway access including retrograde intubation, cricothyrotomy, and tracheotomy is necessary. For providers without advanced surgical training, a cricothyrotomy is easier, associated with less bleeding, and requires less surgical time when compared to tracheotomy [2]. The success rate of cricothyrotomy is between $87 \%-100 \%$ [3] and is an essential tool for any provider who manages the airway.

According to the Goals and Objectives for anesthesia trainees, they must be proficient in all manner of difficult airway management. These objectives include senior residents being able to perform bedside percutaneous tracheotomies. In the emergent setting, regardless of advanced airway training, a cricothyrotomy is preferable.

With this in mind, we set out to assess the knowledge, confidence, and procedural ability of our anesthesia residents in performing a cricothyrotomy. Our hypothesis was that advanced anesthesia training would result in improved performance of a cricothyrotomy and that multimedia education would improve both accuracy and time to completion.

\section{Methods}

Institutional Review Board approval was obtained through the Duke University School of Medicine prior to initiation of the study. Clinical Anesthesia (CA) residents in years 1 through 3 of training were approached in the operative theatre. After successful intubation of their patient, the resident was provided a brief clinical vignette where the only viable option was emergency invasive airway access. They were given a marking pen as if it were a scalpel and asked to perform a cricothyrotomy. Their accuracy and time were documented. They were asked to complete a brief survey. A multimedia educational program including PowerPoint presentation and videos illustrating anatomy, procedural details, and alternate procedures like retrograde intubation was provided to all anesthesia residents, regardless of their decision to participate in the study. At least 4 weeks later, the residents were again presented with the opportunity to participate.

Descriptive statistics were computed for the distance of the markings, demographics of the subject, and education questions.

Correspondence to: Russel Kahmke, Division of Otolaryngology, Head and Neck Surgery, Department of Surgery, Duke University Health System, Durham, NC, USA, DUMC 2824, Durham, NC 27710, USA, Tel: 919-681-6588; Fax: 919668-3103; E-mail: russel.kahmke@dm.duke.edu

Received: March 03, 2015; Accepted: March 21, 2015; Published: March 23 2015 
Paired t-tests were used to compare the pre- and post-intervention answers to the survey for continuous variables, and McNemar's test were used for categorical variables. Statistical analysis was performed using JMP, version 11, SAS Institute Inc., Cary, NC, USA.

\section{Results}

Nine Clinical Anesthesia residents (six CA-1, three CA-3) consented to participate during the pre-intervention period while 18 consented during the post-intervention period (ten CA-1, five CA-2, three CA-3).

During the survey period, participants were asked to rank their knowledge and confidence of complex airway management on a 5 -point Likert scale (Table 1). 67\% believed they received adequate difficult airway training while half of those (33\%) believed they received adequate invasive airway training. Those same participants (33\%) felt comfortable performing an invasive airway procedure. $100 \%$ of participants were interested in receiving additional invasive airway training.

Participants were also asked whether they had received prior training in or performed a variety of airway management techniques (Table 2).

Due to the wide range of providers who routinely manage and perform procedures on airways, $78 \%$ of participants believed Anesthesiologists should have the training and expertise to perform an invasive airway procedure, like a cricothyrotomy, when indicated (Table 3).

All participants performed a horizontal incision prior to intervention. All participants performed a vertical incision after

Table 1. Survey on knowledge and confidence of invasive airway interventions, Likert scale $1-5$.

\begin{tabular}{|l|c|}
\hline & Mean (Range) \\
\hline Knowledge of airway anatomy & $3.4(3-4)$ \\
\hline Confidence with airway anatomy & $3.3(2-4)$ \\
\hline Knowledge of ASA difficult airway pathway & $3.7(3-5)$ \\
\hline Comfort with ASA difficult airway pathway & $3.5(3-5)$ \\
\hline Knowledge of indications for cricothyrotomy & $3.3(2-5)$ \\
\hline Knowledge in performing a cricothyrotomy & $2.8(1-5)$ \\
\hline Confidence with performing cricothyrotomy & $2.1(1-3)$ \\
\hline
\end{tabular}

Table 2. Assessment of previous training and performance of airway securement techniques (LMA, laryngeal mask airway).

\begin{tabular}{|l|c|c|}
\hline & Previously educated \% & Previously performed \% \\
\hline Face mask ventilation & 100 & 100 \\
\hline Orotracheal intubation & 100 & 100 \\
\hline Nasotracheal intubation & 78 & 56 \\
\hline Routine LMA & 100 & 100 \\
\hline Video-assisted laryngoscopy & 100 & 100 \\
\hline LMA for emergency ventilation & 100 & 78 \\
\hline Intubating stylets & 78 & 78 \\
\hline Intubating LMA & 100 & 89 \\
\hline Awake intubation & 78 & 78 \\
\hline Fiberoptic intubation & 89 & 89 \\
\hline Retrograde intubation & 22 & 0 \\
\hline Rigid bronchoscopy & 0 & 0 \\
\hline Intratracheal jet ventilation & 67 & 33 \\
\hline Cricothyrotomy & 56 & 22 \\
\hline Tracheotomy & 56 & 33 \\
\hline
\end{tabular}

Table 3. Specialties appropriate to perform invasive airway procedures (PA, physician's assistant, NP, nurse practitioner).

\begin{tabular}{|l|c|}
\hline Anesthesiology & $78 \%$ \\
\hline General surgery & $89 \%$ \\
\hline Otolaryngology & $100 \%$ \\
\hline Thoracic Surgery & $78 \%$ \\
\hline Intensive care physician & $66 \%$ \\
\hline Pulmonology & $45 \%$ \\
\hline Emergency medicine & $78 \%$ \\
\hline Mid-level provider (PA, NP) & $22 \%$ \\
\hline Paramedic & $45 \%$ \\
\hline
\end{tabular}

intervention. The average weight and BMI of the patients used was not statistically different between the pre- and post-intervention groups. During the pre-intervention period, the cricothyrotomy was on average $0.66 \mathrm{~cm}$ from the middle of the cricothyroid membrane while it was $0.33 \mathrm{~cm}$ in the post-intervention group. This was not statistically significant $(\mathrm{p}<0.167)$. Patient weight and experience level did not affect the results.

The time to perform the cricothyrotomy in the pre-intervention group was 16.75 seconds and 7.5 seconds in the post-intervention group. This was statistically significant $(\mathrm{p}<0.0034)$. For all participants, in patients with a BMI $<25$, the time to perform a cricothyrotomy was significantly improved (13.1 seconds to 6.7 seconds, $\mathrm{p}<0.03)$. In patients with a BMI $>25$ (classified as overweight), the time to perform a cricothyrotomy also significantly improved (21.2 seconds to 7.9 seconds, $\mathrm{p}<0.01)$. Regardless of experience level, the procedural time was significantly faster for overweight patients $(\mathrm{BMI}>25)$ compared to normal weight patients $(\mathrm{p}<0.05)$. For junior level residents $(\mathrm{CA}-1$ and CA-2), the time to perform a cricothyrotomy significantly improved from 16.3 seconds to 7.6 seconds $(\mathrm{p}<0.026)$ and senior level residents (CA-3) also significantly improved from 17.6 seconds to 7.1 seconds $(p<0.0017)$. Level of experience was not statistically significant.

\section{Discussion}

The American Society of Anesthesiologists Task Force on Management of the Difficult Airway published updated practice guidelines in 2013 [1]. In "cannot intubate, cannot ventilate" situations where emergency non-invasive airway ventilation fails, emergency invasive airway access including surgical or percutaneous airway, jet ventilation, or retrograde intubation are necessary. There are personal and contextual reasons for why Anesthesiologists hesitate to obtain a surgical airway including availability of appropriate equipment, personality, and patient presentation [4]. On average about $60 \%$ of Anesthesiologists have experience in "cannot intubate, cannot ventilate" situation and most have little experience and feel uncomfortable with open surgical airways [5]. In an era of trainee work hour restrictions, there is emerging evidence that these restrictions negatively impact medical education and increases complication rates among highacuity patients [6]. Hands on training and simulation has previously been shown to increase comfort and efficiency with difficult airway management [5,7-9]. Considering this evidence, our hypothesis was that the emergent airway procedure performance amongst anesthesia trainees at our institution would improve after a brief multimedia education intervention.

At our tertiary care center, the participating anesthesia trainees were educated and/or had previously performed the most common techniques required to secure an airway before our intervention. However, emergency invasive airway access techniques including 
retrograde intubation, intratracheal jet ventilation, rigid bronchscopy, and surgical airway access were taught less frequently (0-67\%) and performed even less $(0-33 \%)$. Only $78 \%$ of trainees believed that they should have the appropriate expertise to perform an invasive airway procedure while Otolaryngology (100\%) and General surgery (89\%) were believed to be more appropriate providers of a surgical airway. A lack of practical knowledge or technical skill could play a significant role in a lack of perceived expertise [10].

Our results suggest that a lack of practice rather than anatomical knowledge in cricothyrotomy may exist amongst anesthesia trainees. Trainees in the pre-intervention group were not significantly further from the cricothyroid membrane than those in the post-intervention group. Level of expertise, junior residents (CA-1 and CA-2) vs. senior residents, did not have a statistical effect on cricothyrotomy location or time to completion. As demonstrated in the literature, Anesthesiologists with greater than one year of experience did not perform any better in simulator training with increasing levels of experience [11]. This consistency parallels the didactic education received early in anesthesia residency training. However, in a controlled setting with unlimited time, one would expect a high degree of accuracy. Our results indicated that dedicated multimedia education, even without simulation, led to a statistically significant reduction in the time needed to obtain a cricothyrotomy. This effect was more pronounced for overweight or obese individuals with a $\mathrm{BMI}>25$. While these results seem obvious, there is no identifiable literature to support this claim.

A strength of this study is that the subjects receiving a "cricothyrotomy" were actual patients with variant anatomy in a true anesthesia setting. A limitation is that the trainees did not actually perform a cricothyrotomy in either a simulator or ona mannequin. Importantly, this research identified a need for additional invasive airway training amongst anesthesia trainees. As the diagnostic accuracy of predicting a difficult airway situation is quite low [12], anesthesia trainees should feel knowledgeable and confident in all means of obtaining an appropriate airway for all individuals.

\section{Conclusion}

Overall, we found both a knowledge and confidence deficit in emergency invasive airway management. While resident experience level did not contribute to accuracy or time to completion, resident ability to more rapidly perform a cricothyrotomy in overweight patients was noted after intervention. This research highlights a need for dedicated invasive airway training among anesthesia trainees.

\section{References}

1. Apfelbaum JL, Hagberg CA, Caplan RA, Blitt CD, Connis RT, et al. (2013) Practice guidelines for management of the difficult airway: an updated report by the American Society of Anesthesiologists Task Force on Management of the Difficult Airway. Anesthesiology 118: 251-270. [Crossref]

2. Dillon JK, Christensen B, Fairbanks T, Jurkovich G, Moe KS (2013) The emergent surgical airway: cricothyrotomy vs. tracheotomy. Int J Oral Maxillofac Surg 42: 204208. [Crossref]

3. Gillespie MB, Eisele DW (1999) Outcomes of emergency surgical airway procedures in a hospital-wide setting. Laryngoscope 109: 1766-1769. [Crossref]

4. Greenland KB, Acott C, Segal R, Goulding G, Riley RH, et al. (2011) Emergency surgical airway in life-threatening acute airway emergencies--why are we so reluctant to do it? Anaesth Intensive Care 39: 578-584. [Crossref]

5. Wong DT, Lai K, Chung FF, Ho RY (2005) Cannot intubate-cannot ventilate and difficult intubation strategies: results of a Canadian national survey. Anesth Analg 100: 1439-1446. [Crossref]

6. Ahmed N, Devitt KS, Keshet I, Spicer J, Imrie K, et al. (2014) A systematic review of the effects of resident duty hour restrictions in surgery: impact on resident wellness, training, and patient outcomes. Ann Surg 259: 1041-1053. [Crossref]

7. You-Ten KE, Bould MD, Friedman Z, Riem N, Sydor D, et al. (2014) Cricothyrotomy training increases adherence to the ASA difficult airway algorithm in a simulated crisis: a randomized controlled trial. Can J Anaesth 62: 485-494. [Crossref]

8. Wong DT, Prabhu AJ, Coloma M, Imasogie N, Chung FF (2003) What is the minimum training required for successful cricothyroidotomy?: a study in mannequins. Anesthesiology 98: 349-353. [Crossref]

9. Friedman Z, You-Ten KE, Bould MD, Naik V (2008) Teaching lifesaving procedures: the impact of model fidelity on acquisition and transfer of cricothyrotomy skills to performance on cadavers. Anesth Analg 107: 1663-1669. [Crossref]

10. Rosenstock C, Østergaard D, Kristensen MS, Lippert A, Ruhnau B, et al. (2004) Residents lack knowledge and practical skills in handling the difficult airway. Acta Anaesthesiol Scand 48: 1014-1018. [Crossref]

11. Byrne AJ, Jones JG (1997) Responses to simulated anaesthetic emergencies by anaesthetists with different durations of clinical experience. Br J Anaesth 78: 553-556. [Crossref]

12. Norskov AK, Rosenstock CV, Wetterslev J, Astrup G, Afshari A, et al. (2015) Diagnostic accuracy of anaesthesiologists' prediction of difficult airway management in clinica practice: a cohort study of 188064 patients registered in the Danish Anaesthesia Database. Anaesthesia 70: 272-281. [Crossref]

Copyright: (C2015 Kahmke R. This is an open-access article distributed under the terms of the Creative Commons Attribution License, which permits unrestricted use, distribution, and reproduction in any medium, provided the original author and source are credited. 